\title{
Polymorphism and molecular characteristics of the CSN1S2 gene in river and swamp buffalo
}

\author{
Xinyang Fan ${ }^{1} \star$, Shanshan Gao ${ }^{1,}$, Lin Fu ${ }^{1, \star}$, Lihua Qiu ${ }^{1}$, and Yongwang Miao ${ }^{1}$ \\ ${ }^{1}$ Faculty of Animal Science and Technology, Yunnan Agricultural University, Kunming 650201, Yunnan, China \\ These authors contributed equally to this work.
}

Correspondence: Yongwang Miao (yongwangmiao1@126.com)

Received: 20 May 2020 - Revised: 22 July 2020 - Accepted: 4 August 2020 - Published: 18 September 2020

\begin{abstract}
The $\alpha_{S 2}$-casein $\left(\alpha_{S 2}-\mathrm{CN}\right)$ is a member of the casein family associated with milk traits in ruminants, but so far the buffalo CSN1S2 gene has not been well understood. In this work, the polymorphisms of CSN1S2 in river and swamp buffalo were detected using direct sequencing of polymerase chain reaction (PCR) products. As a result, 13 single nucleotide polymorphisms (SNPs) were identified in the coding sequence (CDS) of CSN1S2 in two types of buffalo, of which eight SNPs were non-synonymous. The amino acid changes caused by c.580T $>\mathrm{C}$ and c. $642 \mathrm{C}>\mathrm{G}$ may affect the function of buffalo $\alpha_{S 2}-\mathrm{CN}$. A total of 11 CSN1S2 CDS haplotypes were defined, and accordingly 11 variants of buffalo $\alpha_{S 2}$-CN were inferred and named. The CSN1S2 CDSs of both types of buffalo were 669 nucleotides, which encoded a precursor of 222 amino acids (AAs), and the first 15 AAs constitute a signal peptide. The composition and physicochemical characteristics of two types of buffalo $\alpha_{S 2}$-CNs were similar but slightly different from those of cattle $\alpha_{S 2}$-CN. The $\alpha_{S 2}-\mathrm{CN}$ mature peptides of buffalo and the species of $B o s$ genus contained a casein domain, and their secondary structures were highly consistent, indicating that they are functionally similar. The results here provide initial insights into the variation, characteristics and biological function of buffalo CSN1S2.
\end{abstract}

\section{Introduction}

Casein $(\mathrm{CN})$ is the main protein in milk, accounting for about $80 \%$ of milk protein, including $\alpha_{S 1^{-}}, \alpha_{S 2^{-}}, \beta$ - and $\kappa$-CN. It can provide the suckling infant with amino acids, calcium and phosphate and is related to lactation traits of dairy animals and milk processing characteristics (Boettcher et al., 2004; Wedholm et al., 2006; Nilsen et al., 2009). The CSN1S2 gene encodes $\alpha_{S 2}-\mathrm{CN}$, which accounts for $10 \%$ of the total $\mathrm{CN}$ in the milk of dairy cows (Farrell et al., 2004). The $\alpha_{S 2}-\mathrm{CN}$, together with $\alpha_{s 1}-\mathrm{CN}$ and $\beta$-CN, is called a calcium-sensitive protein which can form stable micelles with calcium and phosphorus to support bone growth in the young (Lefèvre et al., 2009; Corral et al., 2013). In addition, the CSNIS2 gene is related to the nutritive value of milk (Wedholm et al., 2006).

The cattle CSNIS2 gene has been mapped in chromosome 6 and contains 18 exons, of which exon 1 is located in the $5^{\prime}$ untranslated region (UTR), exon 2 encodes the signal pep- tide from the 13th nucleotide, exons 3-16 are located in the coding sequence (CDS) region and encode a mature peptide, and exons $17-18$ are in the $3^{\prime}$ UTR. The CDS length is $669 \mathrm{bp}$ and encodes a protein consisting of 222 amino acids (AAs), of which the first 15 AAs form an $\mathrm{N}$-terminal signal peptide (Farrell et al., 2004). The $\alpha_{S 2}$-CN variants in the Bos genus have been widely studied for many years. So far, five $\alpha_{S 2}-$ $\mathrm{CN}$ variants (A, B, C, D and E) have been identified in the Bos genus (Caroli et al., 2009; Gallinat et al., 2013). Among them, variant $\mathrm{A}$ is the most prevalent and is regarded as the reference protein. Only two $\alpha_{S 2}-\mathrm{CN}$ alleles, $\mathrm{A}$ and $\mathrm{B}$, have been reported in buffalo (Cosenza et al., 2009).

As a kind of dairy, meat and draft animal, the water buffalo (Bubalus bubalis) has important economic value in countries of tropical and subtropical areas (Michelizzi et al., 2010). According to the morphological and behavioral criteria, domestic buffalo can be divided into swamp buffalo and river buffalo. The former is mainly used for draft, while the latter is mainly used for milk production. In the past few years, buf- 
falo have provided about $13 \%$ of the total milk production in the world (Basilicata et al., 2017). In addition, buffalo milk has physicochemical characteristics different from cow milk. Compared with the milk of dairy cattle, buffalo milk has a higher content of total solids, fat and protein (Ahmad et al., 2013). Similarly, buffalo milk has been identified as containing the $\alpha_{S 2}-\mathrm{CN}$ component encoded by the CSN1S2 genes which are located on chromosome 7 (Iannuzzi et al., 2003). Up to now, little research has been done on the CSN1S2 gene in buffalo at the molecular level. In view of the lack of information about this gene, we detected the single nucleotide polymorphisms (SNPs) in the CDS of the CSN1S2 gene for two types of buffalo using direct sequencing of polymerase chain reaction (PCR) products. The $\alpha_{S 2}-\mathrm{CN}$ variants in two types of buffalo were characterized, and the differences of $\alpha_{S 2}-\mathrm{CN}$ variants between buffalo and the species of Bos genus were further investigated. The results can provide a basis for revealing the molecular characteristics, function and variation of the buffalo CSN1S2 gene.

\section{Materials and methods}

\subsection{Animal source and sample collection}

Ear tissue samples were obtained from 120 river buffalo (74 Binglangjiang, 32 Murrah and 14 Nili-Ravi buffalo) and 284 swamp buffalo (58 Xilin, 42 Yanjin, 72 Dechang, 52 Fuzhong and 60 Guizhou buffalo). The buffalo used for sample collection were all healthy adult buffalo without direct kinship. To further align with buffalo, the published CDS sequences of the CSN1S2 gene in the Bos genus including Bos taurus, Bos grunniens, Bos mutus and Bison bison in the National Center for Biotechnology Information (NCBI) database (https: //www.ncbi.nlm.nih.gov/, last access: 10 May 2020) were downloaded and used for data analysis in this study.

All procedures for sample collection were performed in accordance with the Guide for Animal Care and Use of Laboratory Animals approved by the Yunnan Provincial Experimental Animal Management Committee under contract 2007-0069.

\subsection{DNA extraction, PCR and sequencing}

Genomic DNA was isolated from the ear tissue following a protocol described by Sambrock and Russell (2001). Their quality was detected by using $1.5 \%$ agarose gel and further quantified using a NanaDrop LITE spectrophotometer (Thermo Fisher Scientific, USA). Subsequently, six pairs of primers were designed to amplify the CDS of the buffalo CSN1S2 gene according to the genome sequence of buffalo CSN1S2 (accession no. NC_037551) by Primer Premier 5.0 (Table 1) (Lalitha 2000). The $25 \mu \mathrm{L}$ reaction system consisted of $0.6 \mu \mathrm{M}$ of each primer, $100 \mathrm{ng}$ of DNA template and $12.5 \mu \mathrm{L}$ of $2 \times$ GoldStar MasterMix (dye) (CWBio, China). The PCR protocol was performed according to the manufac- turers' instructions of $2 \times$ GoldStar MasterMix (dye). Then the amplified product was electrophoresed on agarose gel, and the target band was purified by cutting gel recovery and further sequenced bidirectionally using the Sanger method.

\subsection{Sequence data analysis}

The obtained sequences of buffalo CSN1S2 were compared, proofread and edited through the Lasergene software package (DNASTAR Inc., USA). Mutation sites were exported with MEGA 6 (Tamura et al., 2013), and estimation of allele and genotype frequencies and Hardy-Weinberg equilibrium test were carried out adopting PopGen32 (Yeh and Boyle, 1997). The function influence of non-synonymous substitutions was presumed using PROVEAN (http://provean.jcvi. org/index.php, last access: 10 May 2020). The haplotypes were analyzed by PHASE (Stephens et al., 2001). The genetic relationship among the haplotypes was constructed by Network 5 (http://www.fluxus-engineering.com, last access: 8 May 2020; Bandelt et al., 1999). The physicochemical characteristics, signal peptide and subcellular localization of buffalo $\alpha_{S 2}-\mathrm{CN}$ were predicted using the ProtParam tool (http://web.expasy.org/protparam/, last access: 10 May 2020), SignalP 5.0 server (http://www.cbs.dtu.dk/ services/SignalP/, last access: 10 May 2020) and ProtComp 9.0 (http://linux1.softberry.com/berry.phtml, last access: 10 May 2020), respectively. The phosphorylation site was presumed through the NetPhos 3.1 Server (http://www. cbs.dtu.dk/services/NetPhos/, last access: 8 May 2020). The conserved domains of buffalo $\alpha_{S 2}-\mathrm{CN}$ were ascertained through the Conserved Domain Architecture Retrieval Tool in BLAST (http://www.ncbi.nlm.nih.gov/BLAST, last access: 10 May 2020). The inferred secondary structure of amino acid sequence was determined by SOPMA (http:// npsa-pbil.ibcp.fr/, last access: 10 May 2020).

\section{Results}

\subsection{Polymorphism analysis of the buffalo CSN1S2 gene}

The PCR products as expected were obtained (Fig. 1). The obtained sequences were assembled and confirmed by comparing them with the homologous sequences of the Bos genus published in NCBI database. In the samples of this work, three SNPs were found in the buffalo CSN1S2 gene, in which c. $234 \mathrm{C}>\mathrm{A}$ was located in exon 9 , c.391G $>\mathrm{A}$ in exon 11 and c.568G $>\mathrm{A}$ in exon 16 (Table 2) with the exception that c.568G $>$ A was only found in river buffalo and the other SNPs were shared by two types of buffalo. It is noteworthy that allele frequency in river buffalo at SNP234 was markedly different from that in swamp buffalo. SNP234 was nearly homozygous in river buffalo, but the heterozygote frequency was still high in swamp buffalo. The test for HardyWeinberg equilibrium showed that only SNP234 in swamp buffalo was in disequilibrium $(P<0.05)$ (Table 2$)$, indicat- 
Table 1. Primer information for PCR and polymorphism identification.

\begin{tabular}{llrrr}
\hline $\begin{array}{l}\text { Amplified } \\
\text { region }\end{array}$ & Primer sequence $\left(5^{\prime}\right.$ to $\left.3^{\prime}\right)$ & $\begin{array}{r}\text { Products } \\
\text { length }(\mathrm{bp})\end{array}$ & $\begin{array}{r}\text { Annealing } \\
\text { temperature }\left({ }^{\circ} \mathrm{C}\right)\end{array}$ & $\begin{array}{r}\text { Extension } \\
\text { time }(\mathrm{s})\end{array}$ \\
\hline Exon 2 & $\begin{array}{l}\text { F: TATGCCCAAATGAGCCTCCA } \\
\text { R: TCCCTCTCTATTCCCTGCTGTC }\end{array}$ & 427 & 53 & 30 \\
\hline Exon 3-5 & $\begin{array}{l}\text { F: TGCCATCAAAACAAACAGGA } \\
\text { R: TGTGGCTCAAAAATGGCTC }\end{array}$ & 1279 & 50 & 105 \\
& F: TTGAGAGCCATTTTTGAGCC & 1628 & 51 & 125 \\
\hline Exon 6-8 & R: GCTCACCCTATTTGCGATGT & & 52.5 & 105 \\
\hline Exon 9-12 & $\begin{array}{l}\text { F: AATGAATTGCCCTTTCTACTC } \\
\text { R: TTCCCCAGATTTTTCTTAGG }\end{array}$ & 1369 & 50 & 25 \\
\hline Exon 13 & $\begin{array}{l}\text { F: GCATTTAGCCAGCATTATG } \\
\text { R: ATCTTACCATGTCAACGGTCT }\end{array}$ & 220 & & 120 \\
\hline Exon 14-16 & F: TTACTGGTGGGCTATTCAAGT \\
& R:CAATTTCCAGCCTAGAACATTC & 1584 & 52.5 & \\
\hline
\end{tabular}

ing that the SNP may be affected by selection and genetic drift.

Having pooled the data of this work with published buffalo sequences (accession numbers FM865618, DW007991, DW007964, DW007983, FM865619 and DQ173244) in the NCBI database, 10 additional SNPs were found in river buffalo, i.e., c. $15 \mathrm{~T}>\mathrm{C}$, c. $381 \mathrm{~T}>\mathrm{A}$, c. $382 \mathrm{~A}>\mathrm{G}$, c. $459 \mathrm{C}>\mathrm{T}$, c. $484 \mathrm{~T}>\mathrm{A}$, c. $580 \mathrm{~T}>\mathrm{C}$, c. $587 \mathrm{~A}>\mathrm{G}$, c. $618 \mathrm{G}>\mathrm{A}$, c. $627 \mathrm{~T}>\mathrm{C}$ and $c .642 \mathrm{C}>\mathrm{G}$, and the number of polymorphic sites increased to 13 . Among them, c. $234 \mathrm{C}>\mathrm{A}, \mathrm{c} .382 \mathrm{~A}>\mathrm{G}$, c. $391 \mathrm{G}>\mathrm{A}$, c. $484 \mathrm{~T}>\mathrm{A}$, c. $568 \mathrm{G}>\mathrm{A}$, c. $580 \mathrm{~T}>\mathrm{C}$, c. $587 \mathrm{~A}>\mathrm{G}$ and c. $642 \mathrm{C}>\mathrm{G}$ were non-synonymous, leading to changes in p.Glu63Asp, p.Lys113Glu, p.Ala116Thr, p.Phe147Ile, p.Ala175Thr, p.Tyr179His, p.Lys181Arg and p.Asn199Lys in the mature peptide (Table S1 in the Supplement). The prediction showed that the substitutions of p.Tyr179His and p.Asn199Lys probably affected the function of $\alpha_{S 2}-\mathrm{CN}$.

\subsection{Haplotype inference and their genetic relationship}

According to the SNPs in the CDS of the buffalo CSN1S2 gene, a total of 11 haplotypes (B1-B11) were inferred in two types of buffalo (Figs. 2 and 3). Among them, five (B1-B5) (accession numbers MT316464-MT316468) were obtained from the data of this study (Table 3), and the other six were from published data (accession numbers FM865618, DW007991, DW007964, DW007983, FM865619 and DQ173244). Among these haplotypes, B1, B3 and B4 were shared by two types of buffalo, B5 was found only in swamp buffalo, and the rest were only found in river buffalo. B6 and B10 (deletion of 27 nucleotides) were equivalent to the previously reported alleles A and B (Cosenza et al., 2009).

The possible genetic relationships among these haplotypes of buffalo CSN1S2 were investigated through employing median-joining network (Fig. 2). Haplotype B1 was the dominant haplotype which was widely distributed in two types of buffalo. Other haplotypes may originate from B1, which is to say that B2, B3 and B10 may evolve from B1 through one transition and $\mathrm{B} 4$ through one transversion. B5 may evolve from B4 through one transition or B3 through a transversion from A to C. B6 may evolve from B2 through a transversion from $\mathrm{T}$ to $\mathrm{A} . \mathrm{B} 7, \mathrm{~B} 8, \mathrm{~B} 9$ and $\mathrm{B} 11$ may evolve from B6 through transitions and/or transversions. Whether this is the case or not, further experiments are needed to verify it.

\subsection{Nucleotide differences of the haplotypes between buffalo and the species of Bos genus}

All the haplotype sequences of the Bos genus published were downloaded and compared with this work in order to explore the differences in the CDS of the CSN1S2 gene between buffalo and the species of Bos genus. A total of 25 haplotypes were defined in the Bos genus (Fig. 3). The comparison results showed that there were 10 differences in the all haplotypes between buffalo and the species of Bos genus, which were located at positions c.49, c. 130 , c. 175 , c. 183 , c. 423 , c.516, c.554, c.572, c.601 and c. 626 .

\subsection{Nomenclature of buffalo $\alpha_{S 2}-\mathrm{CN}$ variants}

A total of $11 \alpha_{S 2}-\mathrm{CN}$ variants were identified in view of the haplotype sequences of buffalo CSN1S2. According to the existing nomenclature of the Bos genus, we named buffalo $\alpha_{S 2}-\mathrm{CN}$ variants as $\mathrm{A}, \mathrm{B}, \mathrm{C}, \mathrm{D}, \mathrm{E}, \mathrm{F}$, G, H, I, J and K (Table 4). Sequence alignment showed that all the variants of the Bos genus identified previously have not been found in two types of buffalo, and there are eight amino acid differences between buffalo $\alpha_{S 2}$ $\mathrm{CN}$ variants and bovine $\alpha_{S 2}-\mathrm{CN}$ variants (Fig. 4), which 

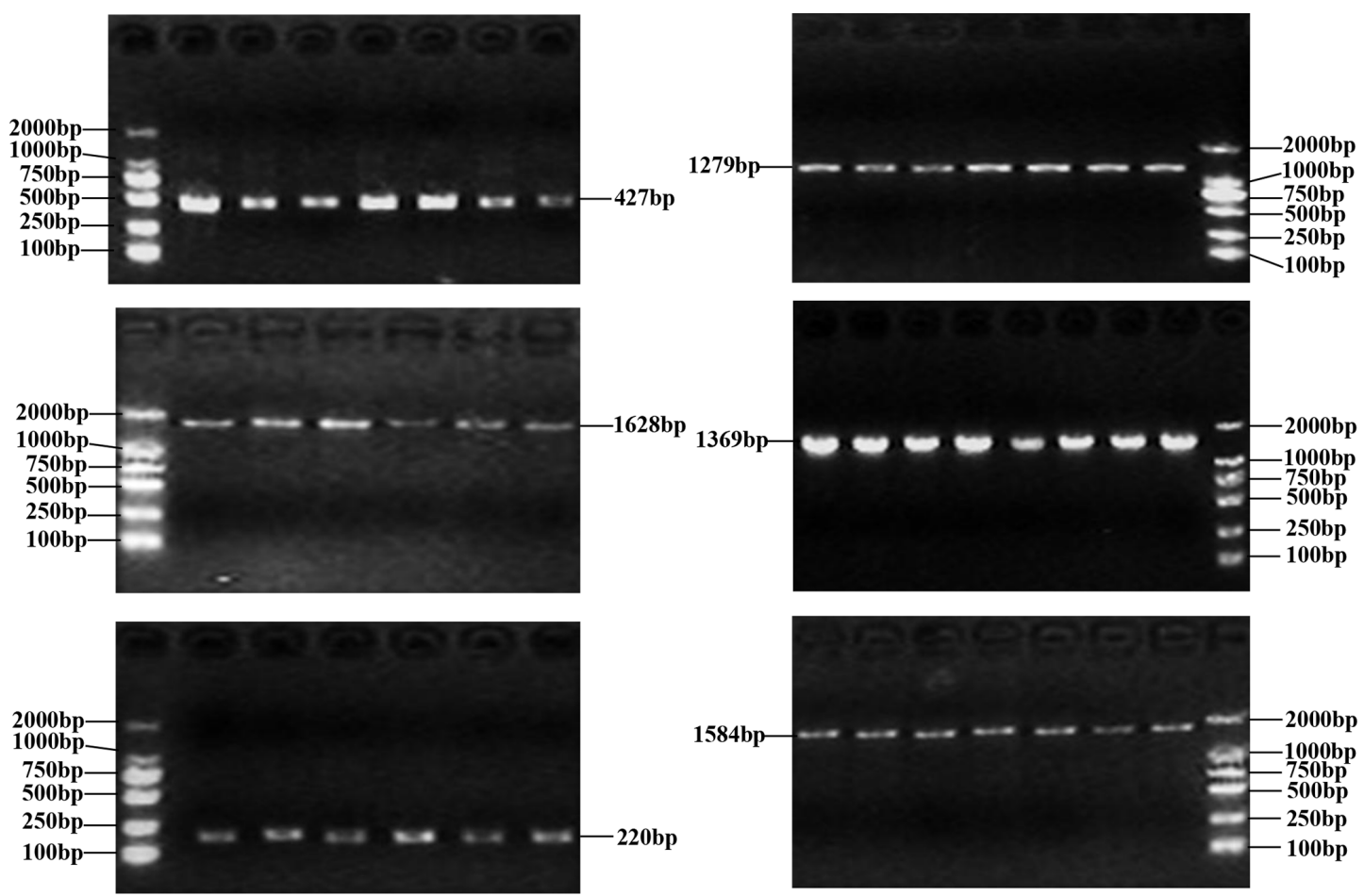

Figure 1. Six PCR-amplified fragments of buffalo CSN1S2 detected by agarose gel electrophoresis.

Table 2. Polymorphic loci and their allelic and genotypic frequencies in two types of buffalo.

\begin{tabular}{|c|c|c|c|c|c|c|}
\hline Population & SNP & \multicolumn{2}{|c|}{$\begin{array}{l}\text { Genotype } \\
\text { frequency }\end{array}$} & \multicolumn{2}{|c|}{$\begin{array}{c}\text { Allele } \\
\text { frequency }\end{array}$} & $P$ value* \\
\hline \multirow[t]{9}{*}{ River buffalo } & c. $234 \mathrm{~A}>\mathrm{C}$ & AA & 0.983 & A & 0.9915 & 1.00000 \\
\hline & & $\mathrm{AC}$ & 0.017 & $\mathrm{C}$ & 0.0085 & \\
\hline & & $\mathrm{CC}$ & 0.000 & & & \\
\hline & c. $391 \mathrm{G}>\mathrm{A}$ & GG & 0.948 & G & 0.9741 & 0.86943 \\
\hline & & GA & 0.052 & A & 0.0259 & \\
\hline & & AA & 0.000 & & & \\
\hline & c. $568 \mathrm{G}>\mathrm{A}$ & GG & 0.339 & G & 0.5893 & 0.85779 \\
\hline & & GA & 0.500 & A & 0.4107 & \\
\hline & & $\mathrm{AA}$ & 0.161 & & & \\
\hline \multirow[t]{6}{*}{ Swamp buffalo } & c. $234 \mathrm{~A}>\mathrm{C}$ & AA & 0.248 & A & 0.4204 & 0.00164 \\
\hline & & $\mathrm{CA}$ & 0.345 & $\mathrm{C}$ & 0.5796 & \\
\hline & & $\mathrm{CC}$ & 0.407 & & & \\
\hline & c. $391 \mathrm{G}>\mathrm{A}$ & GG & 0.558 & G & 0.7458 & 0.86842 \\
\hline & & GA & 0.375 & A & 0.2542 & \\
\hline & & AA & 0.067 & & & \\
\hline
\end{tabular}

* $P$ value of Hardy-Weinberg equilibrium test.

include p.His2Asn, p.His29Asn, p.Ile44Val, p.Asp157Glu, p.His170Arg, p.Trp176Leu, p.Tyr186His and p.Thr194Ile. The sequence consistency of the $\alpha_{S 2}$-CN variants among buffalo and the species of Bos genus was more than $93.0 \%$ (Fig. S1 in the Supplement). In the samples of this work, the frequencies of the variants $\mathrm{A}, \mathrm{B}, \mathrm{C}$ and $\mathrm{D}$ in river buffalo were $58.3 \%, 38.3 \%, 2.5 \%$ and $0.8 \%$, and the frequencies of the variants A, C, D and E in swamp buffalo were $37.3 \%$, $6.3 \%, 41.2 \%$ and $15.1 \%$, respectively. 
Table 3. Frequencies of CSN1S2 haplotypes in two types of buffalo.

\begin{tabular}{llrrrr}
\hline $\begin{array}{l}\text { Haplotype } \\
\text { ID }\end{array}$ & $\begin{array}{l}\text { Base composition } \\
\text { of haplotype }\end{array}$ & $\begin{array}{r}\text { Actual } \\
\text { frequency }\end{array}$ & $\begin{array}{r}\text { Expected } \\
\text { frequency }\end{array}$ & AFR & AFS \\
\hline B1 & AGG & 0.4356 & 0.4070 & 0.5833 & 0.3732 \\
B2 & AGA & 0.1139 & 0.1183 & 0.3833 & 0.0000 \\
B3 & AAG & 0.0520 & 0.0831 & 0.0250 & 0.0633 \\
B4 & CGG & 0.2921 & 0.2932 & 0.0084 & 0.4121 \\
B5 & CAG & 0.1064 & 0.0982 & 0.0000 & 0.1514 \\
\hline
\end{tabular}

Note that the frequency is estimated by the program PHASE. AFR signifies actual frequency in river buffalo, and AFS signifies actual frequency in swamp buffalo.

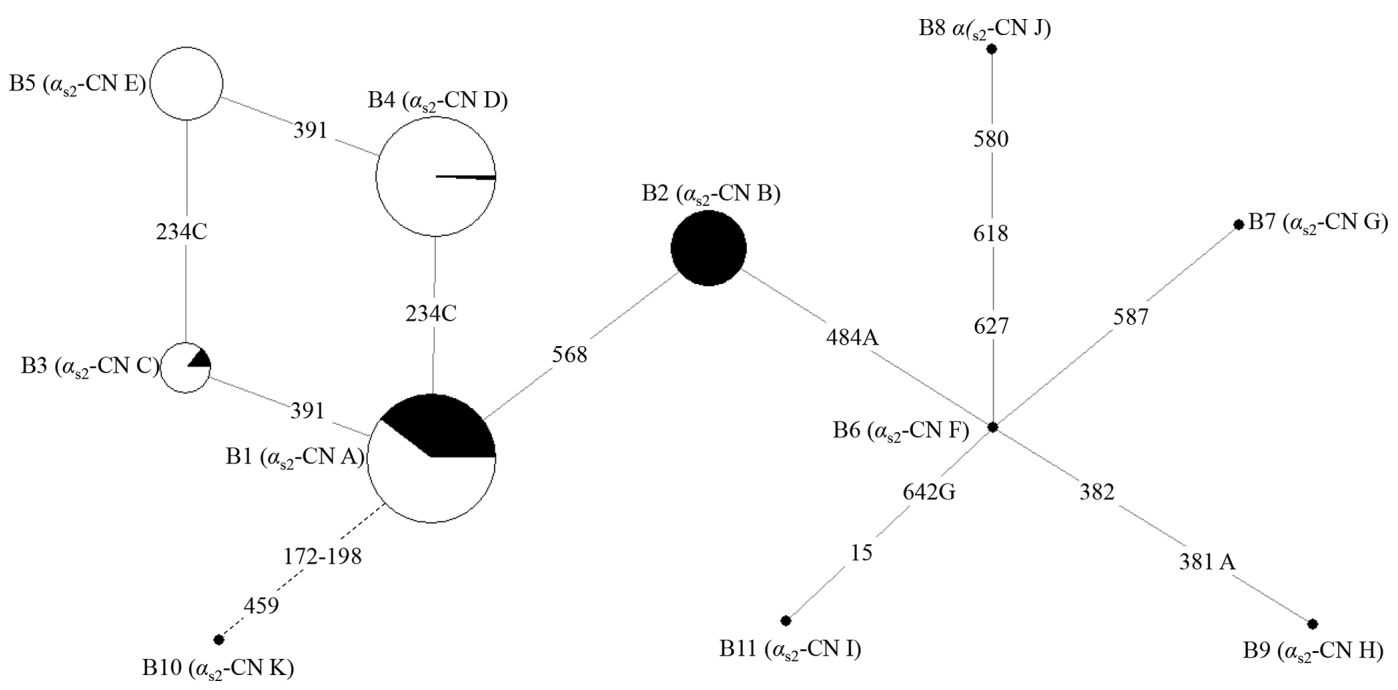

Figure 2. Network of 11 haplotypes of the buffalo CSN1S2 gene. B1-B11 are the haplotypes defined here in buffalo. Mutations along the branch are labeled by the nucleotide positions in the CDS, and transversions are specified by the further addition of suffixes A, G and C. The dotted line represents the haplotype generated from skipping exon 7 after transcription. Each haplotype is represented by a circle with the area of the circle proportional to its frequency. Samples from river and swamp buffalo are indicated by black and white color, respectively.

\subsection{Molecular characteristics analysis}

The length of CSN1S2 CDS for two types of buffalo was 669 nucleotides, encoding a precursor peptide consisting of 222 AAs. Buffalo $\alpha_{S 2}$-CN had a signal peptide of 15 AAs and a mature peptide of 207 AAs. The AA composition of buffalo $\alpha_{S 2}$-CN variant A is shown in Table S2. In order to clarify the characteristics of the buffalo CSN1S2 gene and their differences between buffalo and the species of Bos genus, we analyzed the molecular characteristics of major $\alpha_{S_{2}}-\mathrm{CN}$ variants of buffalo (buffalo variant A from this study, accession no. MT316464) and cattle (variant A; accession no. M94327; Caroli et al., 2009) by bioinformatics methods. The AA composition and basic molecular characteristics of buffalo $\alpha_{S 2}$ $\mathrm{CN}$ variant A were slightly different from those of cattle $\alpha_{S 2}$-CN variant A (Table 5). Their mature peptides all contained a casein domain (from the AA125 to AA196) (Fig. 4), which belonged to the casein family. The secondary structure of buffalo variant $\mathrm{A}$ and cattle variant $\mathrm{A}$ were also similar (Fig. S2). There were only a few differences between them.
The prediction of subcellular localization showed that both buffalo and cattle $\alpha_{S 2}$-CNs were secreted extracellularly with high reliability $(100 \%)$.

\section{Discussion}

In recent years, the polymorphisms of milk proteins have aroused great research interest because the genotypes of milk proteins may be related to milk composition and milk yield of dairy cattle (Nilsen et al., 2009). In this work, the SNPs of the CSN1S2 gene in two types of buffalo were investigated. As a result, 13 SNPs were determined in buffalo. The allele frequency of river buffalo at SNP234 was significantly different from that in swamp buffalo, and SNP568 found in river buffalo has been homozygous in swamp buffalo, which indicated that the variation of the CSNIS2 gene in two types of buffalo had different population genetic characteristics. It was found that two non-synonymous substitutions in buffalo, i.e., c. $580 \mathrm{~T}>\mathrm{C}$ and c. $642 \mathrm{C}>\mathrm{G}$, led to amino acid changes in p.Tyr179His and p.Asn199Lys which seriously affected the 


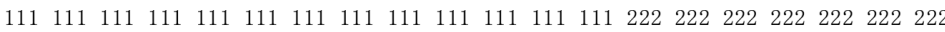

B1
B2
B3
B4
B5
B6
B7
B8
B9
B10
B11

Hap_1 (Bos taurus)

Hap_2 (Bos taurus)

Hap_3 (Bos taurus)

Hap_4 (Bos taurus)

Hap 5 (Bos taurus)

Hap_6 (Bos taurus)

Hap_7 (Bos taurus)

Hap_8 (Bos taurus)

Hap 9 (Bos taurus)

Hap_10 (Bos taurus)

Hap_11 (Bos taurus)

Hap_12 (Bos taurus)

Hap_13 (Bos taurus)

Hap_14 (Bos taurus)

Hap 15 (Bos taurus)

Hap_16 (Bos taurus)

Hap_17 (Bos taurus)

Hap_18 (Bos taurus)

Hap_19 (Bos taurus)

Hap 20 (Bos taurus)

Hap_21 (Bos taurus)

Hap_22 (Bos taurus)

Hap_23 (Bos grunniens)

Hap_24 (Bos mutus)

Hap_25 (Bison bison)

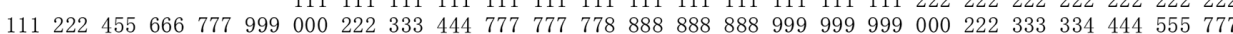
$\begin{array}{lllllllllllllllllllllllllll}345 & 567 & 901 & 789 & 345 & 789 & 678 & 456 & 012 & 234 & 234 & 567 & 890 & 123 & 456 & 789 & 012 & 345 & 678 & 567 & 345 & 234 & 890 & 123 & 012 & 789\end{array}$ ATT CTT CAT TCC AGT GAA AAG GCC CAT GAG GTT ATA AGG AAT GCA AAT GAA GAG GAA ATC GAA GAA GCC ACA GTT TAC
B1
B2
B3
B4
B5
B6
B7
B8
B9
B10
B11

Hap_1 (Bos taurus) Hap_2 (Bos taurus) Hap_3 (Bos taurus) Hap_4 (Bos taurus) Hap_5 (Bos taurus) Hap 6 (Bos taurus) Hap_7 (Bos taurus) Hap_8 (Bos taurus) Hap_9 (Bos taurus) Hap 10 (Bos taurus) Hap 11 (Bos taurus) Hap 12 (Bos taurus) Hap_13 (Bos taurus) Hap 14 (Bos taurus) Hap_15 (Bos taurus) Hap 16 (Bos taurus) Hap 17 (Bos taurus) Hap_18 (Bos taurus) Hap_19 (Bos taurus) Hap 20 (Bos taurus) Hap 21 (Bos taurus) Hap_22 (Bos taurus) Hap_23 (Bos grunniens) Hap_24 (Bos mitus) Hap 25 (Bison bison)

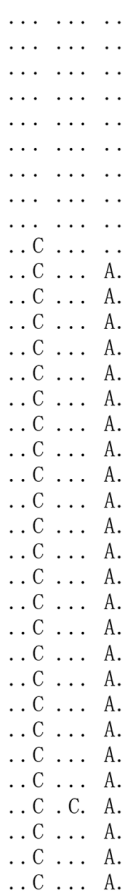

$\begin{array}{lllllllllllllllllllllllll}333 & 333 & 333 & 333 & 333 & 333 & 333 & 444 & 444 & 444 & 444 & 555 & 555 & 555 & 555 & 555 & 555 & 555666 & 666 & 666 & 666 & 666 & 666 & 666 & 666\end{array}$ $\begin{array}{lllllllllllllllllllllllllllll}000 & 111 & 444 & 788 & 888 & 888 & 999 & 222 & 555 & 888 & 999 & 111 & 111 & 555 & 667 & 777 & 888 & 888 & 000 & 000 & 111 & 222 & 444 & 555 & 666 & 666\end{array}$ $\begin{array}{llllllllllllllllllllllllll}789 & 345 & 012 & 901 & 234 & 567 & 123 & 123 & 789 & 456 & 345 & 456 & 789 & 345 & 890 & 123 & 012 & 678 & 123 & 456 & 678 & 567 & 012 & 234 & 123 & 456\end{array}$ TTT CAG CTG GTT AAG AGA GCT GAA ACC TTC AAA GAT AAG CAT GCC TGG TAT AAG TAT CAG AAG ACT AAC TAT TAC CTT

Figure 3. Sequence difference sites of the haplotypes between buffalo and bovine. The corresponding accession numbers of 25 haplotype sequences (Hap1 to Hap25) of the Bos genus are XM_024993017, DR712148, DR711516, DT854174, DR711908, DT849277, DR712290, DR711611, DR711989, EH123804, DR711761, DR71135, M16644, DR711495, DR711941, DR711568, BC114773, DR711392, DR711419, BG690139, BG691827, DR711347, MH378279, XM_014480230 and XM_010852145, respectively. Numbers represent the position in the CDS. Dots (.) represent the identity with the haplotype B1. Nucleotide substitutions are denoted by different letters. Horizontal lines (-) represent the deletion in the sequences. Missing information is denoted by the question marks (?). 
Table 4. Amino acid positions and differences in genetic variants of buffalo $\alpha_{S 2}-\mathrm{CN}$.

\begin{tabular}{|c|c|c|c|c|c|c|c|c|c|c|c|}
\hline \multirow[t]{2}{*}{ Position* } & \multicolumn{10}{|c|}{$\alpha_{S 2}$-CN variant (haplotype) } & \multirow[b]{2}{*}{$\mathrm{K}(\mathrm{B} 10)$} \\
\hline & $\mathrm{A}(\mathrm{B} 1)$ & B (B2) & $\mathrm{C}(\mathrm{B} 3)$ & D (B4) & E (B5) & F (B6) & G (B7) & H (B9) & I (B11) & J (B8) & \\
\hline $43-51$ & & & & & & & & & & & deleted \\
\hline 63 & E GAA & & & D GAC & D GAC & & & & & & \\
\hline 112 & V GTT & & & & & & & GTA & & & \\
\hline 113 & K AAG & & & & & & & E GAG & & & \\
\hline 116 & A GCT & & $\mathrm{T}$ ACT & & $\mathrm{T}$ ACT & & & & & & \\
\hline 138 & $\mathrm{~T}$ ACC & & & & & & & & & & $\mathrm{ACT}$ \\
\hline 147 & F TTC & & & & & I ATC & I ATC & I ATC & I ATC & I ATC & \\
\hline 175 & A GCC & T ACC & & & & T ACC & $\mathrm{T}$ ACC & $\mathrm{T}$ ACC & T ACC & T ACC & \\
\hline 179 & Y TAT & & & & & & & & & H CAT & \\
\hline 181 & K AAG & & & & & & R AGG & & & & \\
\hline 191 & K AAG & & & & & & & & & AAA & \\
\hline 194 & T ACT & & & & & & & & & $\mathrm{ACC}$ & \\
\hline 199 & N AAC & & & & & & & & K AAG & & \\
\hline
\end{tabular}

* Numbers represent the position of the mature peptide.

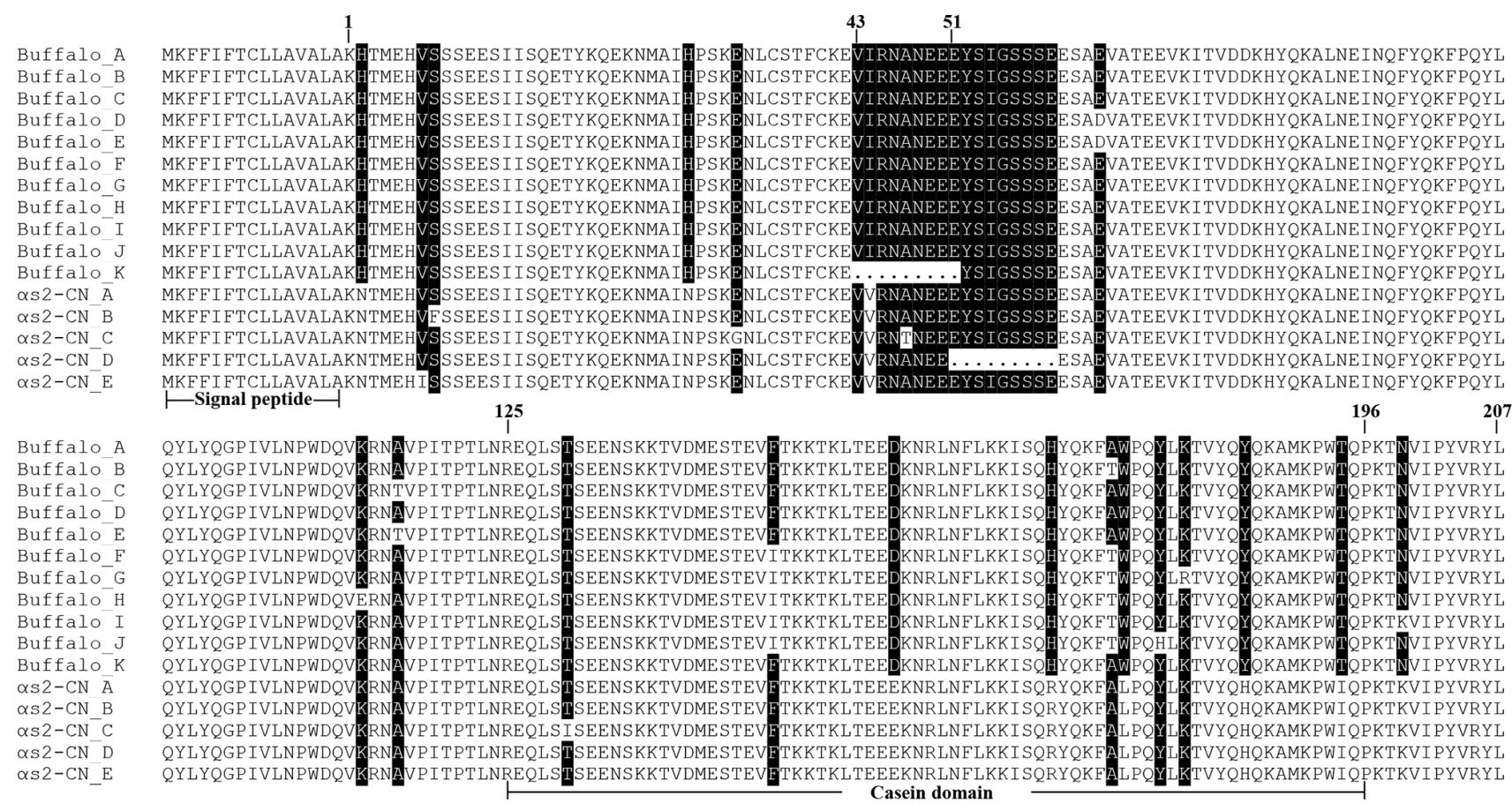

Figure 4. Sequence differences of $\alpha_{S 2}-\mathrm{CN}$ variants between buffalo and the species of Bos genus. $\alpha_{S 2}$-CN_A-E are the identified variant sequences in the Bos genus. Dots (.) represent the deletion in the sequences. Amino acid differences are denoted by black shading.

function of the $\alpha_{S 2}-\mathrm{CN}$. The Tyr and Asn are polar neutral AAs, while the His and Lys are basic AAs. These substitutions belong to substitutions with different physicochemical properties, suggesting that they may cause changes in the structure or function of buffalo $\alpha_{S 2}-\mathrm{CN}$. Whether the SNPs identified in this study, especially the non-synonymous SNPs, have any influence on the function of $\alpha_{S 2}-\mathrm{CN}$ and lactation traits of buffalo needs to be further verified by the association with lactation trait data.
In recent years, the $\alpha_{S 2}-\mathrm{CN}$ variants of the Bos genus have been determined and named (Gallinat et al., 2013). Nevertheless, due to the limited previous studies on the polymorphism of the buffalo CSN1S2 gene, the nomenclature of $\alpha_{S 2}$ $\mathrm{CN}$ variants in buffalo has not been fully developed. In this work, we analyzed the polymorphisms of the CSN1S2 gene in two types of buffalo to achieve a full understanding of buffalo $\alpha_{S 2}-\mathrm{CN}$ variants. It is necessary to separately define the variants of buffalo $\alpha_{S 2}-\mathrm{CN}$ due to the large sequence differ- 
Table 5. Basic physicochemical properties of mature $\alpha_{S 2}-\mathrm{CN}$ peptides from buffalo and cattle.

\begin{tabular}{lrr}
\hline & Buffalo A & Cattle A \\
\hline Formula & $\mathrm{C}_{1091} \mathrm{H}_{1695} \mathrm{~N}_{287} \mathrm{O}_{339} \mathrm{~S}_{6}$ & $\mathrm{C}_{1083} \mathrm{H}_{1707} \mathrm{~N}_{287} \mathrm{O}_{338} \mathrm{~S}_{6}$ \\
Number of amino acids & 207 & 207 \\
Molecular weight & $24.45 \mathrm{KD}$ & $24.35 \mathrm{KD}$ \\
Isoelectric point (pI) & 7.14 & 8.34 \\
Strongly acidic amino acid (D, E) & 28 & 28 \\
Strongly basic amino acid (K, R) & 28 & 30 \\
Polar amino acid (N, C, Q, S, T, Y) & 77 & 76 \\
Hydrophobic amino acid (A, I, L, F, W, V) & 53 & 54 \\
Instability index (II) & 45.64 & 46.27 \\
Grand average of hydropathicity (GRAVY) & -0.944 & -0.918 \\
Aliphatic index & 65.41 & 68.70 \\
Number of amino acids in signal peptide & 15 & 15 \\
Number of phosphorylation sites & 30 & 29 \\
\hline
\end{tabular}

ences of the CSN1S2 gene between buffalo and the species of Bos genus. We named 11 variants in buffalo $\alpha_{S 2}-\mathrm{CN}$ based on the nomenclature convention based on buffalo CSN1S2 haplotypes. In previous studies, it has been reported that there are two kinds of CSN1S2 transcripts in river buffalo, one covering seven exons with a CDS length of $669 \mathrm{bp}$ and the other having an exon 7 skipping (variant $\mathrm{K}$ in this work) with a CDS length of only 642 bp (Cosenza et al., 2009). In this work, we screened SNPs at the DNA level; thus, only the former CSN1S2 transcript was identified in both types of buffalo. It is worth noting that the $\alpha_{S 2}-\mathrm{CN}$ variants with the highest frequency in the two types of buffalo were different. The distribution frequency of variant $A$ is the highest in river buffalo, while the distribution frequency of variant $\mathrm{D}$ is the highest in swamp buffalo.

According to the network of buffalo CSN1S2 haplotypes, $\alpha_{S 2}-\mathrm{CN}$ variants $\mathrm{B}-\mathrm{D}$ differ from variant A by one AA variation, and they may directly evolve from variant A. Buffalo variant $\mathrm{E}$ may evolve from variant $\mathrm{C}$ or $\mathrm{D}$ through one $\mathrm{AA}$ exchange, and variant $F$ may evolve from variant $B$ through one AA exchange. Buffalo variants $\mathrm{G}, \mathrm{H}, \mathrm{I}$ and $\mathrm{J}$ evolved from variant $F$ through one AA exchange for variant $G$, one AA exchange with one synonymous substitution for $\mathrm{H}$, one AA exchange for I, and one AA exchange with two synonymous substitutions for J. Variant K may directly originate from A through exon 7 skipping with one synonymous substitution. Haplotypes B6-B11 came from the sequences published in the database. It is worth noting that the variants encoded by these sequences are very different from those determined by the samples in this study.

Previous studies have confirmed that the CSN1N2 gene exerts an important function in milk traits. In cattle, studies have revealed that the CSN1S2 gene is closely related to the milk protein (Aleandri et al., 1990; Ikonen et al., 2001). The variation in the CSN1S2 gene is associated with the synthesis rate of $\alpha_{S 2}-\mathrm{CN}$ in milk (Ibeagha-Awemu et al., 2008). In addition, the function of $\alpha_{S 2}-\mathrm{CN}$ is related to the formation of
CN micelles (Johansson et al., 2009). In this study, the $\alpha_{S 2-}$ $\mathrm{CN}$ mature peptides for both river and swamp buffalo were all composed of 207 AAs, and their basic physicochemical properties and amino acid composition were basically similar but slightly different from those of cattle. The influence of this difference on function needs to be further studied. It is well known that the lactation characteristics of river buffalo and swamp buffalo are distinct. Because the main variants of $\alpha_{S 2-\mathrm{CN}}$ in the two types of buffalo are different, their physicochemical properties are also different. This difference may be one of the reasons for the difference in lactation characteristics between the two types of buffalo. This study showed that the buffalo $\alpha_{S 2}-\mathrm{CN}$ mature peptide contains all 20 amino acids, of which essential amino acids account for $42.4 \%$, indicating that buffalo $\alpha_{S 2}-\mathrm{CN}$ is one of the important sources of amino acids for the suckling calves. The $\alpha_{S 2}-\mathrm{CN}$ mature peptides of buffalo and cattle have a casein domain with the same length and similar amino acid composition, and the percent identities of the sequences were also high, suggesting that the functions of $\alpha_{S 2}-\mathrm{CN}$ in the two species are similar. The prediction showed that the secondary structure of buffalo $\alpha_{S 2}-\mathrm{CN}$ was highly consistent with that of cattle $\alpha_{S 2}-\mathrm{CN}$. Considering the highly consistent molecular characteristics of $\alpha_{S 2}-\mathrm{CN}$ between buffalo and cattle, it can be speculated that buffalo $\alpha_{S 2}-\mathrm{CN}$ also fulfills crucial function in the formation of casein micelles.

Protein phosphorylation is a significant posttranscriptional modification which can regulate the structure and function of milk protein (Li et al., 2012). Phosphorylation is very important for the binding of metal ions to casein micelles. Casein micelle has an effect on milk coagulation and cheese making (Fan et al., 2019). Taken together, the phosphorylation of $\alpha_{S 2-\mathrm{CN}}$ significantly affects the milk processing characteristics. Because the amino acid at position 194 in the mature peptide of buffalo $\alpha_{S_{2}}$-CN was different from that of bovine $\alpha_{S 2}$-CN (buffalo, 194Thr; cattle, 194Ile), the number of putative phosphorylation sites in buffalo was one more than 
in bovines. Consequently, there may be some differences in phosphorylation process of $\alpha_{S 2}$-CN between buffalo and cattle, which may lead to differences in the structure and function. This may lead to differences in processing characteristics between buffalo milk and bovine milk.

\section{Conclusions}

In this work, 13 SNPs were determined in the buffalo CSN1S2 gene. Among them, eight were non-synonymous substitutions. The CSN1S2 gene of river and swamp buffalo had different population genetic characteristics. A total of 11 haplotypes were defined in the buffalo CSN1S2 gene, and accordingly $11 \alpha_{S 2}-\mathrm{CN}$ variants were identified in buffalo. The amino acid composition and physicochemical characteristics of buffalo $\alpha_{S 2}$-CN are slightly different from those of cattle. The functional domain and secondary structure of buffalo $\alpha_{S 2}-\mathrm{CN}$ were similar to those of the Bos genus $\alpha_{S 2}-\mathrm{CN}$. Whether these SNPs have any influence on the milk traits of buffalo needs to be further studied.

Data availability. The original data used in this study are available from the corresponding author upon request.

Supplement. The supplement related to this article is available online at: https://doi.org/10.5194/aab-63-345-2020-supplement.

Author contributions. YM conceived and designed the research. SG performed the material preparation and experiments. XF, SG and LQ performed the data collection and analysis. XF, LF and YM drafted the paper. All authors read and approved the final paper.

Competing interests. The authors declare that they have no conflict of interest.

Financial support. This research has been supported by the National Natural Science Foundation of China (grant nos. 31760659 and 31460582) and the Natural Science Foundation Key Project of Yunnan Province, China (grant nos. 2014FA032 and 2007C0003Z).

Review statement. This paper was edited by Steffen Maak and reviewed by two anonymous referees.

\section{References}

Ahmad, S., Anjum, F. M., Huma, N., Sameen, A., and Zahoor, T.: Composition and physico-chemical characteristics of buffalo milk with particular emphasis on lipids, proteins, minerals, enzymes and vitamins, J. Anim. Plant Sci., 23, 62-74, 2013.
Aleandri, R., Buttazzoni, L. G., Schneider, J. C., Caroli, A., and Davoli, R.: The effects of milk protein polymorphisms on milk components and cheese-producing ability, J. Dairy Sci., 73, 241255, https://doi.org/10.3168/jds.S0022-0302(90)78667-5, 1990.

Bandelt, H. J., Forster, P., and Röhl, A.: Median-joining networks for inferring intraspecific phylogenies, Mol. Biol. Evol., 16, 37-48, https://doi.org/10.1093/oxfordjournals.molbev.a026036, 1999.

Basilicata, M. G., Pepe, G., Sommella, E., Ostacolo, C., Manfra, M., Sosto, G., Pagano, G., Novellino, E., and Campiglia, P.: Peptidome profiles and bioactivity elucidation of buffalo-milk dairy products after gastrointestinal digestion, Food Res. Int., 105, 1003-1010, https://doi.org/10.1016/j.foodres.2017.12.038, 2017.

Boettcher, P. J., Caroli, A., Stella, A., Chessa, S., Budelli, E., Canavesi, F., Ghi-roldi, S., and Pagnacco, G.: Effects of casein haplotypes on milk production traits in Italian Holstein and Brown Swiss cattle, J. Dairy Sci., 87, 4311-4317, https://doi.org/10.3168/jds.s0022-0302(04)73576-6, 2004,

Caroli, A. M., Chessa, S., and Erhardt, G. J.: Invited review: milk protein polymorphisms in cattle: effect on animal breeding and human nutrition, J. Dairy Sci., 92, 5335-5352, https://doi.org/10.3168/jds.2009-2461, 2009.

Corral, J. M., Padilla, J. A., Izquierdo, M., Martínez-Trancón, M., Parejo, J. C., Salazar, J., and Hernández-García, F. I.: Detection and genetic characterization of ovine CSN1S2B polymorphisms and their associations with milk production traits, Livest. Sci., 153, 10-19, https://doi.org/10.1016/j.livsci.2013.01.008, 2013.

Cosenza, G., Pauciullo, A., Feligini, M., Coletta, A., Colimoro, L., Di Berardino, D., and Ramunno, L.: A point mutation in the splice donor site of intron 7 in the $\alpha_{S 2}$-casein encoding gene of the Mediterranean River buffalo results in an allele-specific exon skipping, Anim. Genet., 40, 791-791, https://doi.org/10.1111/j.1365-2052.2009.01897.x, 2009.

Fan, X., Zhang, Z., Qiu, L., Zhang, Y., and Miao, Y.: Polymorphisms of the kappa casein (CSN3) gene and inference of its variants in water buffalo (Bubalus bubalis), Arch. Anim. Breed., 62, 585-596, https://doi.org/10.5194/aab-62-585-2019, 2019.

Farrell, H. M. J., Jimenez-Flores, R., Bleck, G. T., Brown, E. M., Butler, J. E., Creamer, L. K., Hicks, C. L., Hollar, C. M., Ng-Kwai-Hang, K. F., and Swaisgood, H. E.: Nomenclature of the proteins of cows' milk-sixth revision, J. Dairy Sci., 87, 1641-1674, https://doi.org/10.3168/jds.S00220302(04)73319-6, 2004.

Gallinat, J. L., Qanbari, S., Drögemüller, C., Pimentel, E. C., Thaller, G., and Tetens, J.: DNA-based identification of novel bovine casein gene variants, J. Dairy Sci., 96, 699-709, https://doi.org/10.3168/jds.2012-5908, 2013.

Iannuzzi, L., Di Meo, G. P., Perucatti, A., Schibler, L., Incarnato, D., Gallagher, D., Eggen, A., Ferretti, L., Cribiu, E. P., and Womack, J.: The river buffalo (Bubalus bubalis, $2 n=50$ ) cytogenetic map: assignment of 64 loci by fluorescence in situ hybridization and R-banding, Cytogenet. Genome Res., 102, 6575, https://doi.org/10.1159/000075727, 2003.

Ibeagha-Awemu, E. M., Kgwatalala, P., and Zhao, X.: A critical analysis of production-associated DNA polymorphisms in the genes of cattle, goat, sheep, and pig, Mamm. Genome, 19, 591617, https://doi.org/10.1007/s00335-008-9141-x, 2008. 
Ikonen, T., Bovenhuis, H., Ojala, M., Ruottinen, O., and Georges, M.: Associations between casein haplotypes and first lactation milk production traits in finnish ayrshire cows, J. Dairy Sci., 84, 507-514, https://doi.org/10.3168/jds.s00220302(01)74501-8, 2001.

Johansson, A., Lugand, D., Rolet-Répécaud, O., Mollé, D., Delage, M. M., Peltre, G., Marchesseau, S., Léonil, J., and Dupont, D.: Epitope characterization of a supramolecular protein assembly with a collection of monoclonal antibodies: the case of casein micelle, Mol. Immunol., 46, 1058-1066, https://doi.org/10.1016/j.molimm.2008.09.028, 2009.

Lalitha, S.: Primer Premier 5, Biotech Software \& Internet Report, 1, 270-272, https://doi.org/10.1089/152791600459894, 2000.

Lefèvre, C. M., Sharp, J. A., and Nicholas, K. R.: Characterisation of monotreme caseins reveals lineage-specific expansion of an ancestral casein locus in mammals, Reprod. Fert. Develop., 21, 1015-1027, https://doi.org/10.1071/RD09083, 2009.

Li, S., Wang, J., Wei, H., Yang, Y., Bu, D., Zhang, L., and Zhou, L.: Identification of bovine casein phosphorylation using titanium dioxide enrichment in combination with nano electrospray ionization tandem mass spectrometry, J. Integr. Agr., 11, 439-445, https://doi.org/10.1016/S2095-3119(12)60029-X, 2012.

Michelizzi, V. N., Dodson, M. V., Pan, Z., Amaral, M. E. J., Michal, J. J., McLean, D. J., Womack, J. E., and Jiang, Z.: Water buffalo genome science comes of age, Int. J. Biol. Sci., 6, 333-349, https://doi.org/10.7150/ijbs.6.333, 2010.
Nilsen, H., Olsen, H. G., Hayes, B., Sehested, E., Svendsen, M., Nome, T., Meuwissen, T., and Lien, S.: Casein haplotypes and their association with milk production traits in Norwegian Red cattle, Genet. Sel. Evol., 41, 24, https://doi.org/10.1186/12979686-41-24, 2009.

Sambrock, J. and Russell, D.: Molecular cloning: a laboratory manual, 3rd Edn., Cold Spring Harbor Laboratory Press, Cold Spring Harbor, New York, 6.4-6.12, 2001.

Stephens, M., Smith, N., and Donnelly, P.: A new statistical method for haplotype reconstruction from population data, Am. J. Hum. Genet., 68, 978-989, https://doi.org/10.1086/319501, 2001.

Tamura, K., Stecher, G., Peterson, D., Filipski, A., and Kumar, S.: MEGA6: molecular evolutionary genetics analysis version 6.0, Mol. Biol. Evol., 30, 2725-2729, https://doi.org/10.1093/molbev/mst197, 2013.

Wedholm, A., Hallen, E., Larsen, L. B., Lindmark-Mansson, H., Karlsson, A. H., and Allmere, T.: Comparison of milk protein composition in a Swedish and a Danish dairy herd using reversed phase HPLC, Acta Agr. Scand. A-An., 56, 8-15, https://doi.org/10.1080/09064700600836745, 2006.

Yeh, F. C. and Boyle, T. B. J.: Population genetic analysis of codominant and dominant marker and quantitative traits, Belg. J. Bot., 129, 157-163, 1997. 